\title{
Microscopic and Macroscopic Measurements of Poisson's Ratio of ASTM B557M Using Digital Image Correlation and Local Search Algorithm
}

\author{
Ching-Yuan Chang, ${ }^{*}$ Hui-En Lin, and Dong-Rong Chen \\ Department of Mechanical Engineering, National Taipei University of Technology, Taipei 10617, Taiwan, ROC \\ (Received February 15, 2019; accepted January 19, 2020)
}

Keywords: digital image correlation, in situ measurement, noncontact inspection, real-time inspection, strain concentration, biaxial strain, Poisson's ratio

We have developed a local search algorithm to enhance the computational efficiency of digital image correlation (DIC). This work examined the biaxial strain and Poisson's ratio of a deformed ASTM B557M specimen using a modified model of DIC. We have also developed a model to enable the precise cropping of large images in order to reduce the numerical cost associated with pattern searches within an image stack. The proposed DIC system produces time-displacement curves based on pattern tracking as well as time-strain curves through numerical differentiation. In the region of elastic deformation, the system provides results consistent with those obtained using strain gauges and material testing systems (MTSs). Local deformation at the microscopic scale is captured using a newly developed DIC program, which outputs raw data corresponding to the vertical and horizontal directions. The proposed DIC system yields clear images with $4 \mathrm{~K}$ resolution $(4096 \times 2160$ pixels $)$ and high spatial resolution of $1.9 \mu \mathrm{m}$ based on a program that uses a numerical gradient to locate peaks in correlated results. Specimen preparation is simple, requiring only the application of speckle on the surfaces of featureless objects. The experimental setup requires only one laptop computer and one or more digital cameras from many manufacturers. The proposed DIC program can be embedded within a variety of MTSs, providing precision measurements for a wide variety of applications.

\section{Introduction}

The precision measurement of biaxial strain and Poisson's ratio is crucial for monitoring the mechanical properties of a specimen under tensile testing. Traditional metrologies use two strain gauges associated with the vertical and horizontal directions to measure biaxial strain, from which the Poisson's ratio of deformed specimens is derived. Unfortunately, the strain measurable by a conventional strain gauge is only 2 to $4 \%$, depending on the model type and glue adhesion. The strain values obtained from contact sensors are the average results from corresponding regions, which means that this approach cannot be used to examine microscopic

*Corresponding author: e-mail: chang@mail.ntut.edu.tw

https://doi.org/10.18494/SAM.2020.2679 
deformations in regions of interest (ROIs). Conventional strain gauges mainly measure the elastic deformation and are unable to derive (a) biaxial strain or (b) Poisson's ratio associated with plastic deformations. ${ }^{(1)}$ In contrast, digital image correlation (DIC) is an optical metrology (i.e., noncontact) ideally suited to measurement in industrial applications. ${ }^{(2)}$ The fact that DIC enables multiscale measurement has led to its wide adoption in experimental mechanics, ${ }^{(3)}$ composite structures, ${ }^{(4)}$ and reinforced concrete. ${ }^{(5)}$ Advances in hardware and software have lowered the requirements for the execution of DIC calculations from clusters of highperformance computers to personal and laptop computers. DIC can be implemented using a white light source ${ }^{(6)}$ and is compatible with conventional sensors, such as extensometers, accelerometers, strain gauges, and material testing systems (MTSs). ${ }^{(7)}$

Setting up a DIC experiment is simple. A typical DIC system comprises (a) a specimen with characteristic speckle, (b) digital cameras, (c) a computer, and (d) core algorithms. Speckle patterns on the surface of a sample serve as registered patterns for image tracking. Only polished objects (i.e., those without natural texture) require the application of artificial speckle. DIC systems can be used to measure specimens of various sizes, ranging from $\mu \mathrm{m}$ to $\mathrm{m}$ order, ${ }^{(8)}$ owing to the convenience of cameras with interchangeable lenses (e.g., telephoto, zoom, and macro lenses). Current electronic devices provide high performance and compact size. They can also run multiple threads for parallel processing,${ }^{(9)}$ thereby facilitating the numerical computation [zero-mean normalized cross-correlation (ZNCC)] required for DIC. ${ }^{(10)}$ Pioneering researchers have reduced the mathematical complexity of image correlation and enhanced the computational efficiency of image-tracking algorithms. ${ }^{(1)}$ The DIC program developed in this study uses ZNCC for pixel resolution in conjunction with a numerical gradient to enable subpixel resolution. We developed a mathematical model and modified the core algorithm used for image correlation in order to reduce the computational load of image tracking and enhance the computational performance of image segmentation. As cameras record video segments of an aluminum specimen undergoing controlled deformation, system software converts the video into an image stack. Following the restoration of the images, a graphical user interface (GUI) is used to launch the DIC program in order to derive the biaxial strain and Poisson's ratio at selected points. The proposed DIC system is compatible with a number of MTSs (e.g., MTS Bionix). It can also derive Young's modulus from the measured stress-strain curves.

In the following, we present a brief introduction regarding DIC and the features of precision measurement related to the biaxial strain and Poisson's ratio of large deformations. We also illustrate the benefits of DIC and outline the innovations presented in this study. Section 2 presents a mathematical model of our proposed DIC program, with explanations of the numerical computation of stress, strain, and velocity in the program. Section 3 presents the experimental results obtained from the MTSs, a strain gauge, and the proposed DIC metrology. We demonstrate the high accuracy and high efficiency that can be achieved by this type of modified DIC program. Section 4 shows the advantages of the proposed DIC method, including highly accurate biaxial measurement and highly efficient numerical computation. 


\section{Theoretical Analysis and Mathematical Model of Proposed DIC Program}

The robustness of ZNCC is superior to that of normalized cross-correlation (NCC) owing to the subtraction of the local mean. ZNCC is also highly tolerant to variations in brightness owing to regional normalization. The conventional DIC metrology using ZNCC requires massive numerical computation (addition and multiplication) to locate registered patterns in search regions; however, industrial applications require immediate results following the completion of experiments. In this study, we sought to overcome the computational overhead involved in searching large regions of registered images. In this section, we outline how we achieved the following objectives: (a) downscale the search region of ZNCC and (b) modify the image correlation procedure. The DIC method begins by capturing images and saving the current frame as an image stack. $F(i, j ; k)$ denotes the image intensity (from 0 to 255). In this study, we employed matrix coordinates with the origin located in the top-left corner of images. Symbols $i$ and $j$ respectively denote indexes related to the rows and columns of the images, respectively. Upper case italic symbols denote the size of the indexes. $I$ and $J$ respectively indicate the height and width of the recorded images. $K$ indicates the total number of images, and the size of the registered patterns is $2 L+1$ and $2 M+1$. The mathematical description of the registered patterns gives us the following:

$$
G_{\alpha}(l, m ; k)=F\left(u_{\alpha}(k)+l, v_{\alpha}(k)+m\right),
$$

where $\alpha$ is the index of the registered patterns and $k$ is the frame number counting from zero. $u_{\alpha}(k)$ and $v_{\alpha}(k)$ are the coordinates of the central point of the registered patterns corresponding to node $\alpha . G_{\alpha}(l, m ; 0)$ denotes the image intensity of the registered patterns in their initial state $F(i, j ; 0)$. The semicolon denotes decoupled variables in the spatial and temporal domains. The core algorithm of conventional ZNCC is written as

$$
\begin{gathered}
H_{\alpha}(i, j ; k)=\frac{\sum_{l=-L}^{L} \sum_{m=-M}^{M}[F(i+l, j+m ; k)-\bar{F}(i, j ; k)]\left[G_{\alpha}(l, m ; k)-\bar{G}_{\alpha}(k)\right]}{\overline{\bar{F}}(i, j ; k) \overline{\bar{G}}_{\alpha}(k)}, \\
\bar{F}(i, j ; k)=\frac{1}{(2 L+1)(2 M+1)} \sum_{l=-L}^{L} \sum_{m=-M}^{M} F(i+l, j+m ; k), \\
\bar{G}_{\alpha}(k)=\frac{1}{(2 L+1)(2 M+1)} \sum_{l=-L}^{L} \sum_{m=-M}^{M} G_{\alpha}(l, m ; k), \\
\overline{\bar{F}}(i, j ; k)=\sqrt{\sum_{l=-L}^{L} \sum_{m=-M}^{M}(F(i+l, j+m ; k)-\bar{F}(i, j ; k))^{2}}
\end{gathered}
$$




$$
\overline{\bar{G}}_{\alpha}(k)=\sqrt{\sum_{l=-L}^{L} \sum_{m=-M}^{M}\left(G_{\alpha}(l, m ; k)-\bar{G}_{\alpha}(k)\right)^{2}},
$$

where $H_{\alpha}(i, j ; k)$ is the correlated result, ranging from 0 to 1 . We can abbreviate the conventional computation as $H_{\alpha}(i, j ; k)=F(i, j ; k) \otimes G_{\alpha}(l, m ; k) . \bar{F}(i, j ; k)$ and $\bar{G}_{\alpha}(k)$ are the mean values in the regions proximal to $F(i+l, j+m ; k)$ and $G_{\alpha}\left(u_{\alpha}(k)+l, v_{\alpha}(k)+m\right) ; \overline{\bar{F}}(i, j ; k)$ and $\overline{\bar{G}}_{\alpha}(k)$ are the standard deviations around the local regions $F(i+l, j+m ; k)$ and $G_{\alpha}\left(u_{\alpha}(k)+l, v_{\alpha}(k)+m\right)$, respectively. Retrieving the tracking results of $u_{\alpha}(k+1)$ and $v_{\alpha}(k+1)$ requires the initial values of $u_{\alpha}(k)$ and $v_{\alpha}(k)$, respectively. In this study, we employed fast NCC. ${ }^{(12)}$ The mathematical model of Eq. (2) has been simplified as ${ }^{(13)}$

$$
H_{\alpha}(i, j ; k)=\frac{h_{\alpha}(i, j ; k)-(2 L+1)(2 M+1) \bar{F}(i, j ; k) \bar{G}_{\alpha}(k)}{\overline{\bar{F}}(i, j ; k) \overline{\bar{G}}_{\alpha}(k)} .
$$

The cross-correlation term $h_{\alpha}(i, j ; k)$ can be obtained either in the spatial domain as

$$
h_{\alpha}(i, j ; k)=\sum_{l=-L}^{L} \sum_{m=-M}^{M} F(i+l, j+m ; k) G_{\alpha}(i, j ; k)
$$

or in the frequency domain as

$$
h_{\alpha}(i, j ; k)=\mathrm{F}^{-1}\left\{\mathrm{~F}\{F(i+l, j+m ; k)\} \mathrm{F}\left\{G_{\alpha}(i, j ; k)\right\}\right\} \text {, }
$$

where the nonitalic symbols $\mathrm{F}\{\}$ and $\mathrm{F}^{-1}\{\}$ respectively denote the operator of the Fourier transform and that of the inverse Fourier transform. Equations (8) and (9) yield the same results, differing only in the fact that numerical calculation in the frequency domain becomes increasingly efficient as (a) $L$ approaches $M$ and (b) $L$ and $M$ increase. ${ }^{(13,14)}$ We adopted Eq. (8) because the following experiments adopt small registered patterns. The maximum peak in the correlated result $H_{\alpha}(i, j ; k)$ is used to locate the registered pattern in the search region as

$$
\left[u_{\alpha}(k+1), v_{\alpha}(k+1)\right]=\operatorname{peak}\left\{H_{\alpha}(i, j ; k)\right\},
$$

where $u_{\alpha}(k+1)$ and $v_{\alpha}(k+1)$ indicate decoupled displacements in the vertical and horizontal directions, respectively. The nonitalic symbol peak \{\} denotes the operator used to find peaks. We adopted the gradient method to identify the location of peak $H_{\alpha}(i, j ; k)$ while providing subpixel resolution. ${ }^{(15)}$ The computational results of $u_{\alpha}(k+1)$ and $v_{\alpha}(k+1)$ are inherited from $u_{\alpha}(k)$ and $v_{\alpha}(k)$ and parallel computation is executable only along index $\alpha$, i.e., it is invalid along index $k$. Our program converts the number of frames, $k$, into real time $t$ using the frame rate of the digital camera employed. The conversion coefficient from frame to time has been denoted as $C_{k t}$ and is a constant in this study. The decoupled results of $u_{\alpha}(k)$ and $v_{\alpha}(k)$ can be plotted 
as time-displacement curves in the horizontal and vertical directions, respectively. Numerical differentiation in the time domain provides time-velocity and time-acceleration curves, giving us the following:

$$
\begin{gathered}
\Delta u_{\alpha}(t)=u_{\alpha}(t)-u_{\alpha}(0) ; \Delta v_{\alpha}(t)=v_{\alpha}(t)-v_{\alpha}(0), \\
w_{\alpha}(t)=\sqrt{\left(\Delta u_{\alpha}(t)\right)^{2}+\left(\Delta v_{\alpha}(t)\right)^{2}}=\sqrt{\left(u_{\alpha}(t)-u_{\alpha}(0)\right)^{2}+\left(v_{\alpha}(t)-v_{\alpha}(0)\right)^{2}}, \\
u_{\alpha}^{\prime}(t)=\frac{u_{\alpha}(t+\Delta t)-u_{\alpha}(t)}{\Delta t} ; u_{\alpha}^{\prime \prime}(t)=\frac{u_{\alpha}^{\prime}(t+\Delta t)-u_{\alpha}^{\prime}(t)}{\Delta t}, \\
v_{\alpha}^{\prime}(t)=\frac{v_{\alpha}(t+\Delta t)-v_{\alpha}(t)}{\Delta t} ; v_{\alpha}^{\prime \prime}(t)=\frac{v_{\alpha}^{\prime}(t+\Delta t)-v_{\alpha}^{\prime}(t)}{\Delta t},
\end{gathered}
$$

where $\Delta t$ is the time interval between two sequential images. The corresponding horizontal displacements are denoted as $u_{A}(t)$ and $u_{B}(t)$, and the vertical displacements are denoted as $v_{A}(t)$ and $v_{B}(t)$. The computation of strain requires the selection of the two points $A$ and $B$. The calculation of real length and engineering strain gives us

$$
\begin{gathered}
w_{A B}(t)=\sqrt{\left(u_{A}(t)-u_{B}(t)\right)^{2}+\left(v_{A}(t)-v_{B}(t)\right)^{2}}, A, B \subset \alpha, A \neq B, \\
w_{A B}^{\prime}(t)=\frac{w_{A B}(t+\Delta t)-w_{A B}(t)}{\Delta t} ; w_{A B}^{\prime \prime}(t)=\frac{w_{A B}^{\prime}(t+\Delta t)-w_{A B}^{\prime}(t)}{\Delta t}, \\
\varepsilon_{A B}(t)=\frac{\Delta w_{A B}(t)}{w_{A B}(0)}=\frac{w_{A B}(t)-w_{A B}(0)}{w_{A B}(0)} .
\end{gathered}
$$

Figure 1 presents a flow chart of a conventional DIC program: (a) recording of images in the image stack $F(i, j ; k)$ given the initial values $u_{\alpha}(0)$ and $v_{\alpha}(0)$ by a calibrated camera; (b)

\section{Initialization}

$F(i, j ; k)$
Image contains all
recorded images
$u_{\alpha}(1), v_{\alpha}(1)$
Initial coordinates of
selected points
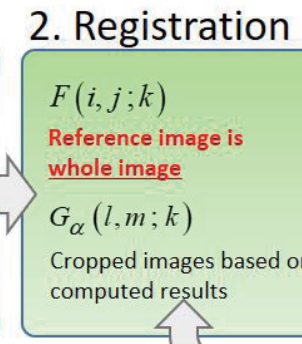

3. Correlation

$F(i, j ; k) \otimes G_{\alpha}(l, m ; k)$
Correlated result based on zero mean cross-correlation

$u_{\alpha}(k), v_{\alpha}(k)$

Biaxial displacement based on peak found by Eq. (10)

\section{Analysis}

$l_{A B}(t), \varepsilon_{A B}(t)$ Length and strain

$u_{\alpha}^{\prime}(t), v_{\alpha}^{\prime}(t)$

$u_{\alpha}^{\prime \prime}(t), v_{\alpha}^{\prime \prime}(t)$

Velocity and acceleration

Iteration

Fig. 1. (Color online) Program flow chart of conventional DIC program: (a) recording experimental image as image stack; (b) cropping registered patterns to form original image stack; (c) iterating ZNCC and retrieving vertical and horizontal displacements; (d) calculating experimental strain and velocity. 
cropping of registered patterns $\mathrm{G}_{\alpha}(l, m ; 0)$ to form the original image stack $F(i, j ; 0)$; (c) iterating ZNCC from Eqs. (7)-(10) and retrieving the decoupled displacement of $u_{\alpha}(k)$ and $v_{\alpha}(k)$; (d) calculating the strain for engineering analysis. In material testing, the MTS generally uses either displacement control or force control. The specimen undergoes steady displacement and the speed of deformation is stable and low. It is not necessary to search for registered patterns in the entire image; therefore, our modification of the program narrows the size of the search region from $I, J$ to $n L, n M$. The default value of $n$ is set at 2 to reduce the computational overhead of ZNCC and may be modified according to the velocity of the tracking results given by Eq. (13). The program crops subset images $F_{\alpha}(\tilde{l}, \tilde{m} ; k)$ to form the initial image stack $F(i, j ; k)$ in accordance with the coefficient $n, u_{\alpha}(k)$, and $v_{\alpha}(k)$, resulting in the following:

$$
F_{\alpha}(\tilde{l}, \tilde{m} ; k)=F\left(u_{\alpha}(k)+\tilde{l}, v_{\alpha}(k)+\tilde{m} ; k\right),
$$

where $\tilde{l}$ ranges from $-n l$ to $n l$ and $\tilde{m}$ ranges from $-n m$ to $n m$. The size of the search region, $F_{\alpha}(\tilde{l}, \tilde{m} ; k)$, is $2 n L+1$ (rows) and $2 n M+1$ (columns). The program separates large images $F(i, j ; k)$ into several small subset images $F_{\alpha}(\tilde{l}, \tilde{m} ; k)$ and $G_{\alpha}(l, m ; k)$, and the index notation changes from global $(i$ and $j)$ to local $(\alpha, l$, and $m$ ). Cropping images reduces the size of the search region and increases the computational efficiency of executing ZNCC, such that

$$
\begin{gathered}
H_{\alpha}(\tilde{l}, \tilde{m} ; k)=\frac{h_{\alpha}(\tilde{l}, \tilde{m} ; k)-(2 L+1)(2 M+1) \bar{F}(\tilde{l}, \tilde{m} ; k) \bar{G}_{\alpha}(k)}{\overline{\bar{F}}(\tilde{l}, \tilde{m} ; k) \overline{\bar{G}}_{\alpha}(k)}, \\
{\left[u_{\alpha}(k+1), v_{\alpha}(k+1)\right]=\left[u_{\alpha}(k), v_{\alpha}(k)\right]+\operatorname{peak}\{H(\tilde{l}, \tilde{m} ; k)\}, \text { where } k=2, \ldots, K .}
\end{gathered}
$$

$H_{\alpha}(\tilde{l}, \tilde{m} ; k)=F_{\alpha}(\tilde{l}, \tilde{m} ; k) \otimes G_{\alpha}(l, m ; k)$ is the abbreviation of the proposed computation. Equations (7) and (10) search for registered patterns in the entire image, whereas Eqs. (19) and (20) search for registered patterns only in local regions. The former method is valid in most general cases, including dynamic impact and transient vibration. The latter method is more efficient in cases of slow displacement or quasi-static deformation. Our modified DIC program requires additional work (image cropping and coordinate transform); however, it minimizes the search region. The coefficient $n$ depends on the strain rate and is adjustable in accordance with the speed of deformation due to an external load. The reduction in computational overhead compensates for the CPU time required to crop images and perform the coordinate transform.

Figure 2 presents a flow chart of the proposed DIC program: (a) retrieving the initial image $F(i, j ; l)$ and assigning initial values for $u_{\alpha}(1), v_{\alpha}(1)$; (b) cropping registered patterns $G_{\alpha}(l, m ; 1)$ to form the original image stack $F(i, j ; 1)$; (c) cropping subset images $F_{\alpha}(\tilde{l}, \tilde{m} ; k)$ from the current image $F(i, j ; k)$ and executing ZNCC using Eqs. (19) and (20); (d) calculating strain and velocity for the subsequent quantitative analysis. The proposed DIC program makes it possible to obtain precise measurements with high efficiency while providing the option to skip the 


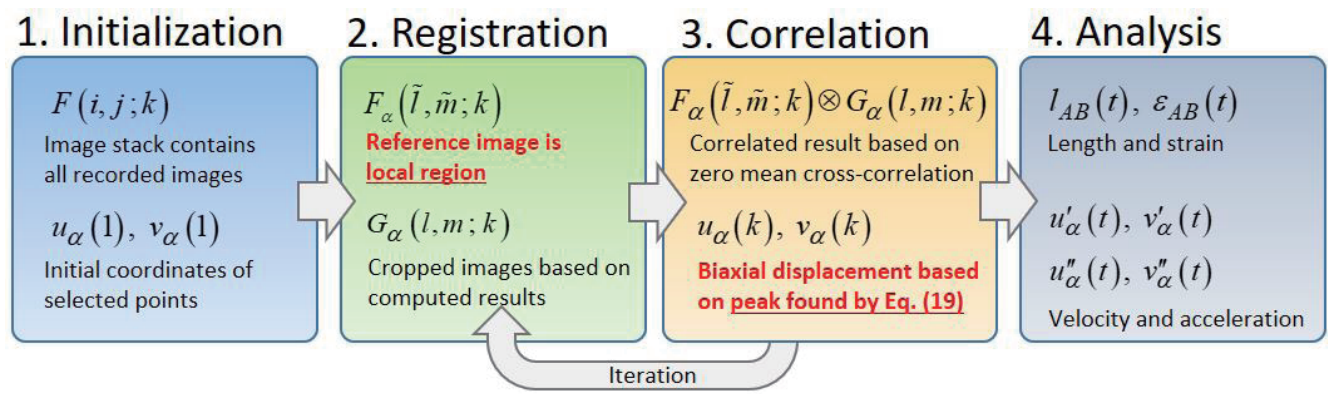

Fig. 2. (Color online) Program flow chart of proposed DIC program: (a) retrieving initial image and setting initial values; (b) cropping registered patterns to form original image stack; (c) retrieving current image and executing ZNCC given by Eqs. (19) and (20); (d) calculating experimental strain and velocity for real-time observation.
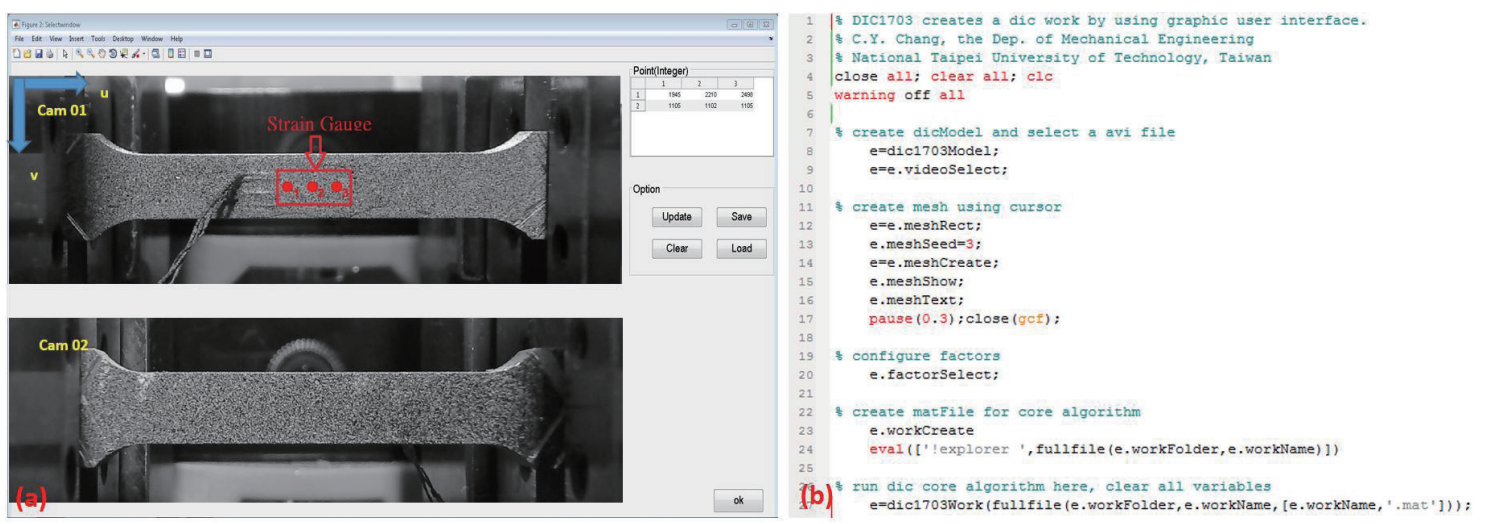

Fig. 3. (Color online) Demonstration of proposed DIC program: (a) GUI and (b) command line for new analysis.

image restore step in order to reduce the computational overhead. This makes it possible for the proposed DIC program to retrieve image pixels directly from memory instead from a hard drive disk. There is also the option to restore the image to a hard drive disk and verify the accuracy of the results using a number of cross-correlation criteria. All of the algorithms in this study were implemented in MATLAB 2015a and embedded in a laptop computer (Intel i7-7500U with 8G DDR4-RAM).

Figure 3 shows the GUI and command lines used to launch DIC analysis. Our proprietary DIC program uses the architecture of object orientation to run the following procedures: (i) basic input/output of images and videos, (ii) calibration of spatial and temporal resolutions, (iii) automatic placement of mesh for regular and irregular boundaries, (iv) manual configuration of $L, M$, and $n$, (v) core algorithm of the proposed DIC method, and (vi) analytical functions used in retrieving the time-displacement and time-strain curves, and Poisson's ratio. Figure 3(a) shows the GUI, which includes dual images obtained from two cameras. Cameras 1 and 2 were respectively focused on the front and back sides of a B557M specimen, following the geometry defined by the American Society for Testing and Materials (ASTM). The GUI interface provides a batch file including all configurations using command lines for the execution of DIC. Figure 3(b) presents the command lines of our DIC program, where line 8 creates a new 
job for the DIC criterion by calling up the self-defined DIC class. In the command line, users can type coordinates to select tracking points, which are precisely the same points as those used for benchmark testing. Our program provides raw data of time-displacement, and time-strain curves, and Poisson's ratio, i.e., without smoothing or filtering. Our program takes advantage of object orientation while providing a high degree of flexibility in adjusting the criteria of image correlation.

\section{Theoretical Analysis and Mathematical Model of Proposed DIC Program}

Figure 4 presents the experimental setup for the tensile testing of ASTM B557M and the DIC experiment. The experiment involved the following steps: (a) attachment of a strain gauge (SHOWA N11-FA-5-350-11-VM5T) to the front of the B557M specimen; (b) spraying speckle on the sample surface to enhance the signal-to-noise ratio; (c) applying pretension to the specimen followed by the calibration of the digital camera, MTS, and oscilloscope; (d) selecting tracking points and launching the proposed DIC program shown in Fig. 2. In this study, we compared the experimental results obtained using the (i) strain gauge system, (ii) MTS, and (iii) DIC system. The strain gauge system was an NI DAQ 9236 system with a maximum sampling rate of 100 points/s. The subscripts SG and MTS denote the measured results obtained from the
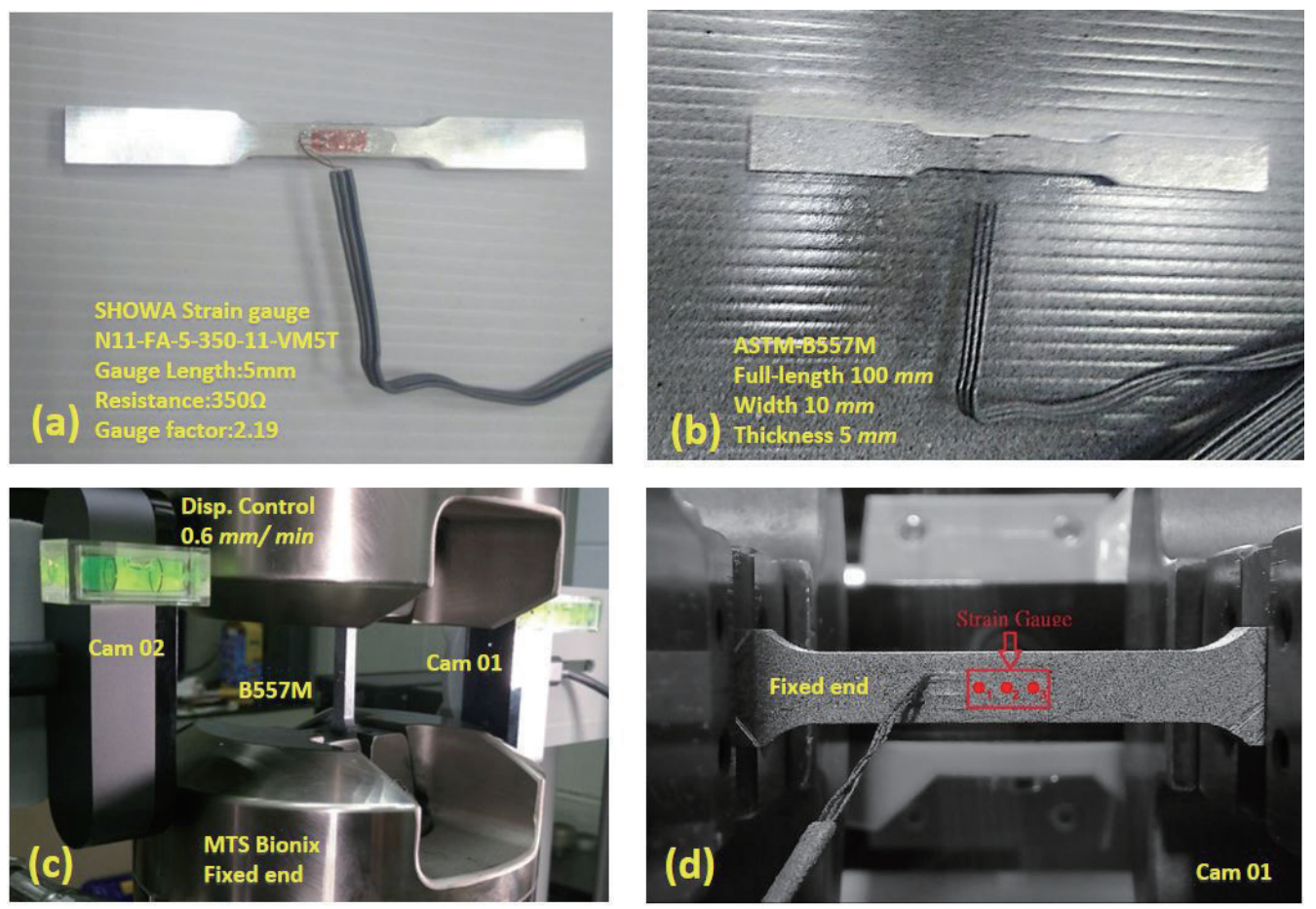

Fig. 4. (Color online) Experimental setup of B557M specimen for tensile test and DIC measurement: (a) attaching strain gauge (SHOWA N11-FA-5-350-11-VM5T) to center of specimen; (b) spraying speckle on specimen surface; (c) applying pretension and calibrating digital camera, MTS, and oscilloscope; (d) selecting observation region and launching real-time DIC program shown in Fig. 2. 
strain gauge and MTS, respectively. The strain obtained using the strain gauge is denoted as $\varepsilon_{S G}(t)$. The size of the SHOWA N11-FA-5-350-11-VM5T strain gauge is $5.0 \times 2.6 \mathrm{~mm}^{2}$. In the MTS, we use the displacement control mode with a speed of $0.6 \mathrm{~mm} / \mathrm{min}$ on the top side of the specimen. The bottom side is fixed. $F_{M T S}(t)$ denotes the external force recorded by the MTS at a sampling rate of 100 points/s. The elongation of the B557M specimen at time $t$ is denoted in terms of $w_{M T S}(t)$ with an initial length $w_{M T S}(0)$ of $45 \mathrm{~mm}$. The area of the cross section is $A_{M T S}(t)$ and the initial area $A_{M T S}(0)$ is $30 \mathrm{~mm}^{2}\left(6 \times 5 \mathrm{~mm}^{2}\right)$. The normal stress applied by the MTS is $\sigma_{M T S}(t)=F_{M T S}(t) / A_{M T S}(0)$, which was previously used to derive stress-strain curves. The DIC system comprises two high-resolution cameras (Logitech BRIO Webcam) producing images with $4096 \times 2160$ pixels.

The camera on the front side of the specimen verifies the measured strain by comparison with those obtained from the strain gauge. The camera on the back side measures the strain on the deformed specimen. Each camera provides time-displacement and time-strain curves. The average frame rate of both cameras was 1.6/s with a temporal resolution of $625 \mathrm{~ms}$. The default values of $L$ and $M$ were both 30 pixels, and the area of the registered pattern was $61 \times 61$ pixels. The physical length of a pixel was $19 \mu \mathrm{m} /$ pixel. Thus, the spatial resolution was estimated to be $1.9 \mu \mathrm{m}$ from subpixel calculations. All of the computed DIC results were converted into actual distances rather than pixels. The total time required to complete the experiment was $538 \mathrm{~s}$ from the initial trigger until the specimen broke. During this period, each camera obtained 860 frames, resulting in an image stack of $3.81 \mathrm{~GB}$.

Figure 5 shows the search region and the computational efficiency of the proposed DIC method. The blue rectangle in Fig. 5(a) indicates the registered pattern and the red rectangle indicates the search region. The size of the registered pattern is $61 \times 61$ pixels, while that of the search region is $121 \times 121$ pixels. The half window of the blue rectangle is 30 pixels and the search window is 60 pixels. In this case, the coefficient of the search region, $n$, is 2. Increasing $n$ was shown to increase the computational overhead of image correlation. Figure 5(b) presents the time required to derive the time-displacement curves using various $n$ values. The computational cost corresponding to different $n$ values in each frame was $T(30,30, n)=0.0025 n^{2}-0.00251 n+0.1237$. To analyze the 860 frames, the average

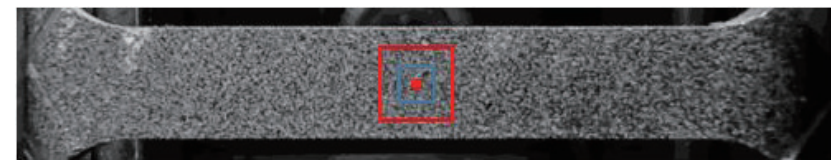

(a)

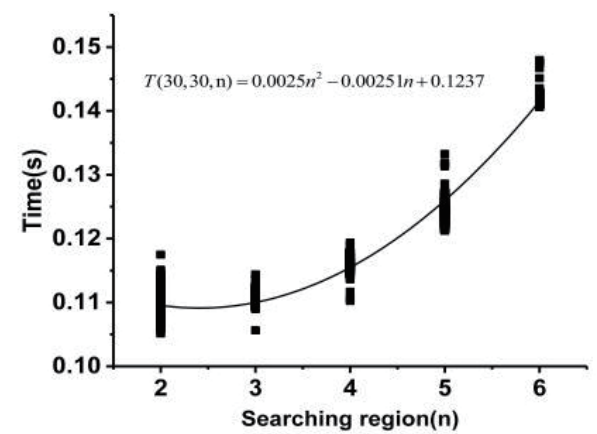

(b)

Fig. 5. (Color online) Benchmark of proposed method: (a) the blue rectangle denotes the registered image and the red rectangle denotes the search region; (b) the computational cost corresponding to different $n$ values. 
computational time of executing the DIC program was considered to be $95.31 \mathrm{~s}$ for $n=2$ and $124.23 \mathrm{~s}$ for $n=6$. The speed up was 1.3 times. Increasing $n$ enlarged the search region, thereby increasing the time required to compute Eqs. (18)-(20). Mechanical deformations implemented at higher speeds resulted in a larger $n$ value and required a larger region for pattern searching. As shown in Fig. 5, $n=2$ achieved good performance with adequate efficiency in the experiment shown in Fig. 4. In the subsequent DIC analysis, we selected a default value of $n=2$ and set the initial half window at 30 pixels.

Figure 6(a) presents the initial images retrieved from cameras 1 and 2. Camera 1 was focused on the front side, while camera 2 was focused on the back side. Deformation was analyzed using points $G, C$, and $S$ on the front side, and the corresponding locations on the back side were $G^{\prime}, C^{\prime}$, and $S^{\prime}$, where the prime symbol denotes that the selected points are on the back side without a strain gauge sensor. Camera 1 measured the strain of the strain gauge and camera 2 inspected the strain on the deformed specimen. Figure 6(a) also shows the fixed and moving ends of the B557M specimen. Figure 6(b) presents the stress-strain curve obtained from the MTS and strain gauge. Young's modulus was measured to be $70.23 \mathrm{GPa}$ and the yielding stress was $265 \mathrm{MPa}$. The strain gauge failed at $75.93 \mathrm{~s}(1.92 \%)$, which is insufficient for the investigation of mechanical behavior under large deformation. Figure 6(c) presents the time-displacement curves obtained using the MTS and DIC method. The slope of the MTS curve was measured at $0.6 \mathrm{~mm} / \mathrm{min}$. The selected points $G, C$, and $S$ were respectively used
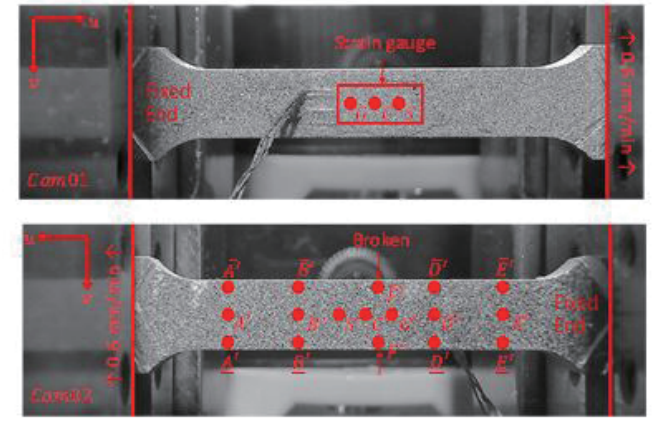

(a)

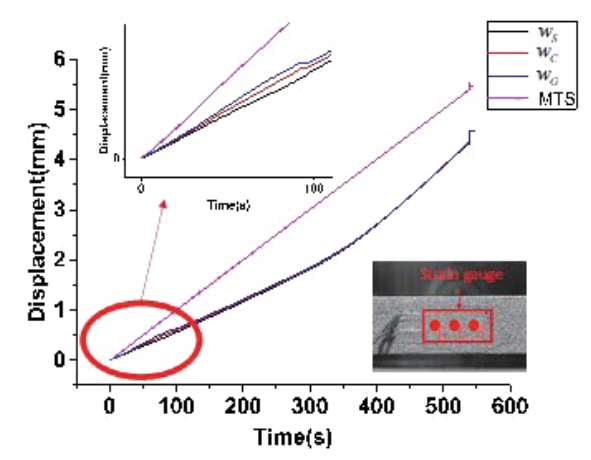

(c)

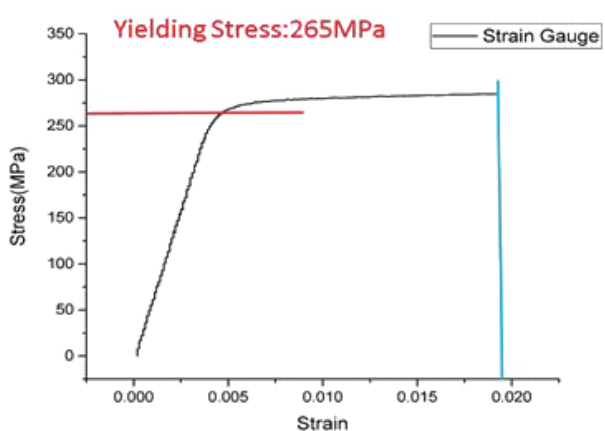

(b)

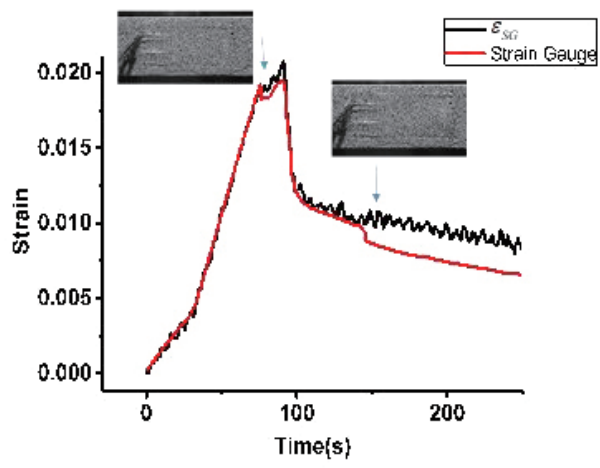

(d)

Fig. 6. (Color online) Experimental results: (a) initial images retrieved from cameras 1 and 2; (b) stress-strain curve obtained from MTS system and strain gauge; (c) time-displacement curves obtained using MTS and DIC method; (d) time-strain curves obtained using strain gauge and DIC method. 
to track the left end, center, and right end on the strain gauge, where $w_{G}(t), w_{C}(t)$, and $w_{S}(t)$ are the corresponding time-displacement results. The displacement of points $G, C$, and $S$ was nonlinear, particularly after the onset of necking at $352 \mathrm{~s}$. Figure 6(d) presents the time-strain curves obtained using the strain gauge and DIC method. Before $75.93 \mathrm{~s}$, the measured timestrain curves obtained from the DIC method and strain gauge are highly consistent; after $75.93 \mathrm{~s}$, the glue between the strain gauge and the B557M specimen gradually lost adhesion.

Figure 7(a) presents the time-displacement curves for points $A^{\prime}, B^{\prime}, C^{\prime}, D^{\prime}, E^{\prime}, S^{\prime}$, and $G^{\prime}$, as well as those obtained from the back of the specimen. The displacement of point $A^{\prime}$ (close to the moving end) exceeded that of point $E^{\prime}$ (near the fixed end). The measured time-displacement curves obtained from $W_{G^{\prime}}(t), W_{C^{\prime}}(t)$, and $W_{S^{\prime}}(t)$ differed from those obtained from $W_{G}(t), W_{C}(t)$, and $W_{S}(t)$ owing to the fact that points $G^{\prime}, C^{\prime}$, and $S^{\prime}$ track mechanical deformation directly on the surface of the specimen, rather than through the strain gauge. At $352 \mathrm{~s}$, strain concentration was combined with necking effects, which resulted in nonlinear deformation, as indicated by the measurements obtained from all of the points. Figure 7(b) presents the time-strain curves obtained using the DIC method. The selected lines were $C^{\prime} G^{\prime}, S^{\prime} G^{\prime}, B^{\prime} D^{\prime}, A^{\prime} E^{\prime}, G^{\prime} E^{\prime}$, and $A^{\prime} S^{\prime}$ with the DIC strain calculated using Eq. (17). Note that $C^{\prime} G^{\prime}$ (close to the location of the final break $\bar{F}^{\prime} \underline{F}^{\prime}$ ) yielded the maximum strain values, exceeding those of $S^{\prime} G^{\prime}, B^{\prime} D^{\prime}, A^{\prime} E^{\prime}, G^{\prime} E^{\prime}$, and $A^{\prime} S^{\prime}$.

The corresponding time-strain relationships were $\varepsilon_{C^{\prime} G^{\prime}}(t), \varepsilon_{S^{\prime} G^{\prime}}(t), \varepsilon_{B^{\prime} D^{\prime}}(t), \varepsilon_{A^{\prime} E^{\prime}}(t), \varepsilon_{G^{\prime} E^{\prime}}(t)$, and $\varepsilon_{A^{\prime} S^{\prime}}(t)$, and the average strain rate was $3185 \mu \varepsilon /$ s prior to the onset of necking. $\max \left\{\varepsilon_{C^{\prime} G^{\prime}}(t)\right\}$, $\max \left\{\varepsilon_{S^{\prime} G^{\prime}}(t)\right\}$, and $\max \left\{\varepsilon_{B^{\prime} D^{\prime}}(t)\right\}$ denote the maximum values of the time-strain curves as follows: $\varepsilon_{C^{\prime} G^{\prime}}(t)(588846 \mu \varepsilon), \varepsilon_{S^{\prime} G^{\prime}}(t)(470554 \mu \varepsilon)$, and $\varepsilon_{B^{\prime} D^{\prime}}(t)(285264 \mu \varepsilon)$. The values of $\max \left\{\varepsilon_{C^{\prime} G^{\prime}}(t) / \varepsilon_{S^{\prime} G^{\prime}}(t)\right\}$ and $\max \left\{\varepsilon_{C^{\prime} G^{\prime}}(t) / \varepsilon_{B^{\prime} D^{\prime}}(t)\right\}$ used to quantify the effects of strain localization were 1.2514 and 2.0642, respectively. The proposed DIC method provides raw data (i.e., without digital filtering), which means that the details pertaining to deformation are preserved. The high degree of linearity observed in the time-displacement curves in the elastic zone demonstrates that the boundary conditions were well controlled.

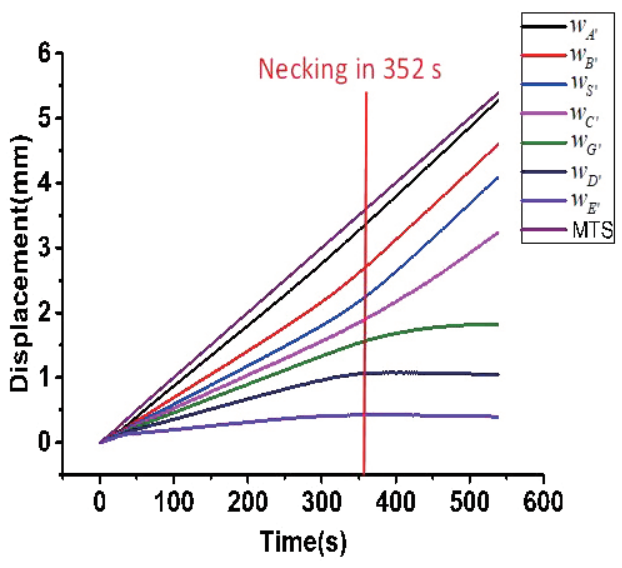

(a)

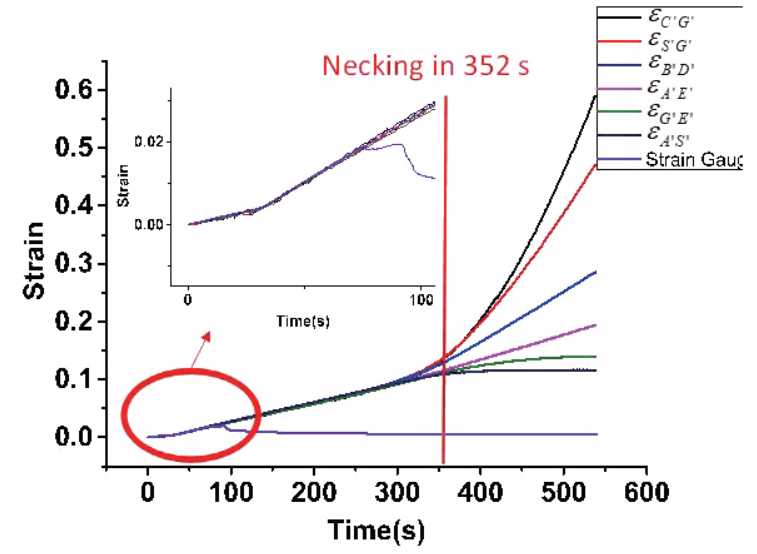

(b)

Fig. 7. (Color online) Raw data obtained from DIC system: (a) time-displacement curve along vertical direction and (b) time-displacement curve along horizontal direction. 
Figure 8(a) presents the time-displacement curves for points $\bar{A}^{\prime}, \underline{A}^{\prime}, \bar{B}^{\prime}, \underline{B^{\prime}}, \bar{D}^{\prime}, \underline{D^{\prime}}, \bar{E}^{\prime}, \underline{E^{\prime}}$, $\bar{F}^{\prime}$, and $\underline{F}^{\prime}$, which were used to inspect the vertical deformation of the B557M specimen. The upper and lower bars of the symbols respectively indicate that the measured point is above or beneath the center point. The section in which the final break occurred was at $\bar{F}^{\prime} \underline{F}^{\prime}$, and the absolute displacements of $w_{\bar{F}^{\prime}}(t)$ and $w_{\underline{F}^{\prime}}(t)$ exhibited a clear increase following the onset of necking. The proposed DIC method provides a spatial resolution of $1.9 \mu \mathrm{m}$, which is sufficient to analyze small deformations in the vertical direction. Figure $8(\mathrm{~b})$ presents the time-strain curves of $\varepsilon_{\bar{A}^{\prime} \underline{A}^{\prime}}(t), \varepsilon_{\bar{B}^{\prime} \underline{B}^{\prime}}(t), \varepsilon_{\bar{D}^{\prime} \underline{\underline{D}}^{\prime}}(t), \varepsilon_{\bar{E}^{\prime} E^{\prime}}(t)$, and $\varepsilon_{\bar{F}^{\prime} \underline{F}^{\prime}}(t)$. Negative strain is an indication of compressive deformation in the vertical direction. At $\varepsilon_{\bar{F}^{\prime} F^{\prime}}(t)$, the average strain rate was determined to be $73845 \mu \varepsilon / \mathrm{s}$ before the onset of necking and $113110 \mu \varepsilon / \mathrm{s}$ after the onset of necking. At $\varepsilon_{\bar{A}^{\prime} \underline{A}^{\prime}}(t)$, the average strain rate was determined to be $74393 \mu \varepsilon / \mathrm{s}$ before the onset of necking (at $352 \mathrm{~s}$ ) and $1290 \mu \varepsilon / \mathrm{s}$ after the onset of necking. The ratio of $\max \left\{\varepsilon_{\bar{F}^{\prime} \underline{F}^{\prime}}(t) / \varepsilon_{\bar{A}^{\prime} \underline{A}^{\prime}}(t)\right\}$ used to quantify the effects of strain concentration was 6.627. These results indicate that the proposed DIC method can be used to measure biaxial displacement as well as inspect deformation at multiple scales.

Figure 9(a) presents the stress-strain curves obtained using the MTS/DIC method. The average Young's modulus obtained using the MTS/DIC method was $70.23 \mathrm{GPa}$ prior to plastic deformation. The measured stresses of yielding and necking were 275 and $305 \mathrm{MPa}$, respectively. The corresponding engineering strains were 0.52 and $0.95 \%$. We also employed an ultrasonic sensor (Olympus 5058PR) and an oscilloscope (LeCory wavesurfer 64X) to measure the material properties of the aluminum specimen before conducting the tensile tests. The velocities of longitudinal and transverse waves were measured to be 6423.10 and 3119.11 $\mathrm{m} / \mathrm{s}$, respectively. The density of the specimen was $2714.98 \mathrm{~kg} / \mathrm{m}^{3}$ and Young's modulus was $71.09 \mathrm{GPa}$ according to ultrasonic measurements. The discrepancy between the values obtained using the MTS/DIC method and those obtained using the ultrasonic method was $-1.2 \%$. Figure 9(b) shows the tracking points during the onset of necking. Figures 9(c) and 9(d) present the measured Poisson's ratio based on Fig. 7(b). Figure 9(c) presents the measured

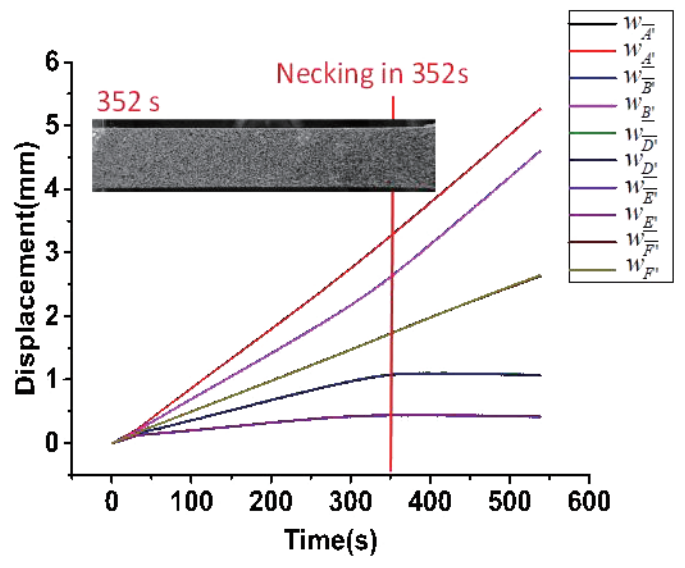

(a)

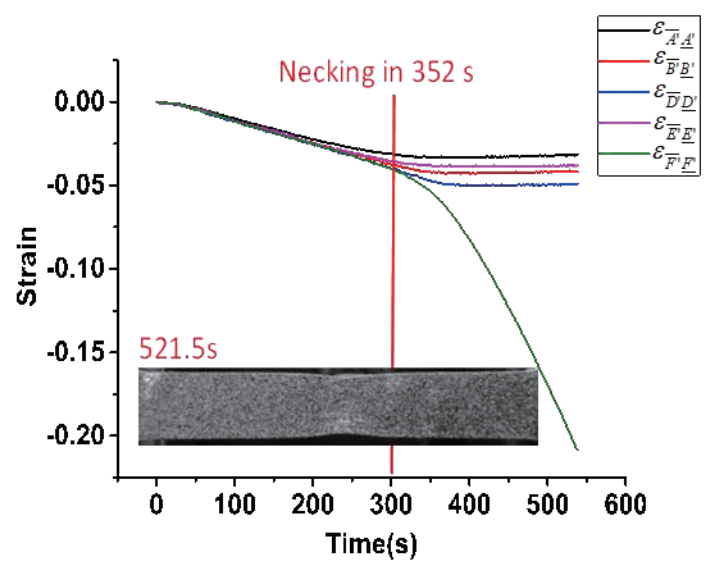

(b)

Fig. 8. (Color online) Biaxial strain based on Fig. 7 and Eq. (17): (a) time-strain curve along vertical direction and (b) time-strain curve along horizontal direction. 


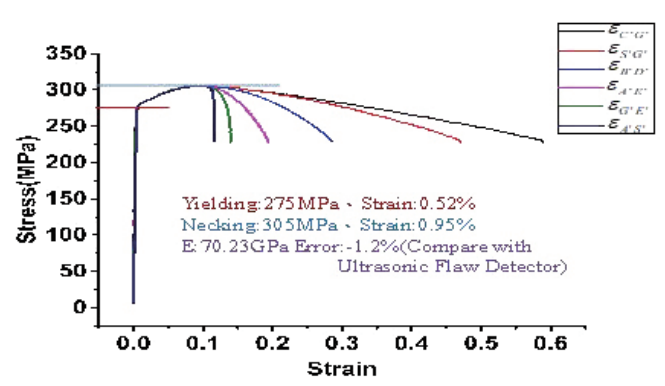

(a)

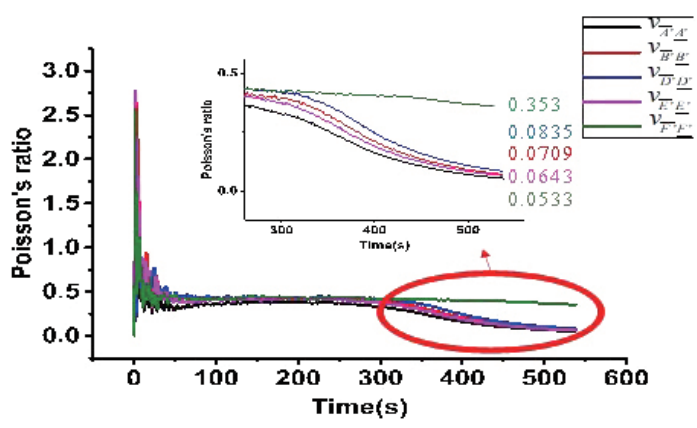

(c)

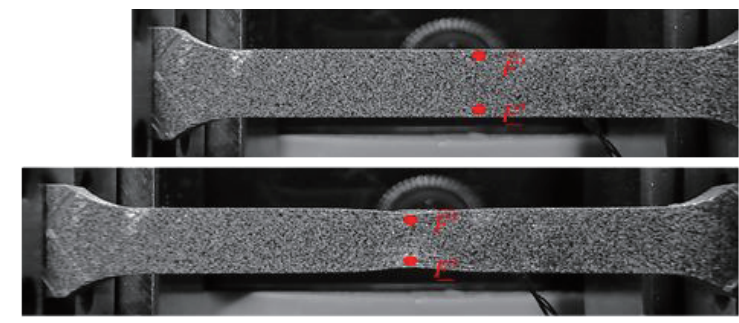

(b)

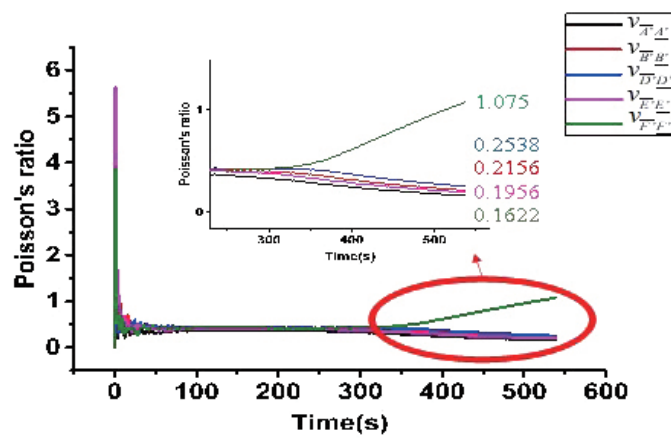

(d)

Fig. 9. (Color online) Young's modulus and Poisson's ratio obtained from experimental measurement: a) stressstrain curves obtained from strain gauge, MTS, and DIC system; (b) tracking points and image of necking effect; (c) time-Poisson's ratio curve obtained from DIC system based on $\varepsilon_{\bar{F}^{\prime} F^{\prime}}(t) / \varepsilon_{C^{\prime} G^{\prime}}(t)$; (d) time-Poisson's ratio curve obtained from DIC system based on $\varepsilon_{\bar{F}^{\prime} \underline{F}^{\prime}}(t) / \varepsilon_{A^{\prime} E^{\prime}}(t)$.

Poisson's ratio $\varepsilon_{\bar{F}^{\prime} \underline{F}^{\prime}}(t) / \varepsilon_{C^{\prime} G^{\prime}}(t)$. The average values before and after necking were 0.426 and 0.381 , respectively. The terminal value of $\varepsilon_{\bar{F}^{\prime} F^{\prime}}(t) / \varepsilon_{C^{\prime} G^{\prime}}(t)$ was 0.344 . The Poisson's ratio in various sections changed after the onset of necking. The Poisson's ratio $\varepsilon_{F_{F^{\prime} \underline{F}^{\prime}}}(t) / \varepsilon_{C^{\prime} G^{\prime}}(t)$ remained stable; however, the values of $\varepsilon_{\bar{A}^{\prime} A^{\prime}}(t) / \varepsilon_{C^{\prime} G^{\prime}}(t), \varepsilon_{\bar{B}^{\prime} B^{\prime}}(t) / \varepsilon_{C^{\prime} G^{\prime}}(t)$, and $\varepsilon_{\bar{E}^{\prime} E^{\prime}}(t) / \varepsilon_{C^{\prime} G^{\prime}}(t)$ decreased after the onset of necking. $\varepsilon_{\bar{A}^{\prime} \underline{A}^{\prime}}(t), \varepsilon_{\bar{B}^{\prime} \underline{B}^{\prime}}(t), \varepsilon_{\bar{D}^{\prime} \underline{D}^{\prime}}(t), \varepsilon_{\bar{E}^{\prime} E^{\prime}}(t)$, and $\varepsilon_{\bar{F}^{\prime} \underline{F}^{\prime}}(t)$ exhibited lower levels of vertical deformation than $\varepsilon_{\bar{F}^{\prime} F^{\prime}}(t)$.

These effects verify the concentration of strain. Figure 9(d) presents the measured Poisson's ratio $\varepsilon_{\bar{F}^{\prime} F^{\prime}}(t) / \varepsilon_{A^{\prime} E^{\prime}}(t)$. The average values were 0.418 before the onset of necking and 0.703 after the onset. The terminal value was 1.08. Note that the Poisson's ratio of a stable, isotropic, linear elastic material is greater than -1.0 and less than 0.5 , and most materials have a Poisson's ratio between 0.0 and 0.5. According to industrial specifications, the value of aluminum 6061 is 0.33 . This high Poisson's ratio can be attributed to the fact that $\varepsilon_{A^{\prime} E^{\prime}}(t)$ is a global result from the entire B557M specimen, whereas $\varepsilon_{F^{\prime} F^{\prime}}(t)$ is a local result. Compared with that of $\varepsilon_{\bar{F}^{\prime} \underline{F}^{\prime}}(t) / \varepsilon_{A^{\prime} E^{\prime}}(t)$, the calculation of Poisson's ratio $\varepsilon_{\bar{F}^{\prime} \underline{F}^{\prime}}(t) / \varepsilon_{C^{\prime} G^{\prime}}(t)$ is better suited to cases of plastic deformation and large deformation. Unfortunately, the region of measurement is less than $4.7 \times 2.4 \mathrm{~mm}^{2}$, and it is difficult to attach two strain gauges in such a small region. Thus, obtaining measurements of biaxial strain in local areas can be simple using conventional strain gauges or extensometers. Well-controlled boundary conditions and a robust algorithm are required to retrieve accurate strain values owing to the fact that numerical differentiation amplifies the noise within the measured displacement. The high degree of consistency in our 
experimental results demonstrates that the proposed DIC method with a local search algorithm is valid for quasi-static deformation. The proposed DIC system enables the inspection of mechanical deformation at the microscopic scale without the need to attach additional sensors. The optical nature of image correlation also avoids the mass effects arising from glue adhesion and provides results of sufficient accuracy to function as a standard test method.

\section{Conclusions}

In this study, we measured Poisson's ratio in a local region using our newly developed DIC program. This DIC program uses a local search algorithm and reduces the computational costs of searches in large images. The front camera retrieved time-strain curves are in good agreement with those obtained using a strain gauge. The numerical precision and experimental reliability of the proposed method were verified via tensile testing. The proposed DIC program provides subpixel resolution based on the algorithm of numerical gradients. Raw data (i.e., without filtering) are used to preserve details pertaining to deformation in the formulation of time-displacement and time-strain curves. Unlike the MTS and strain gauges, the DIC system outlined in this paper measures displacement and strain using one or more cameras and one laptop computer, and is fully compatible with universal MTSs. The proposed DIC system measures biaxial strain and Poisson's ratio without the need for additional sensors. The measured Young's modulus is consistent with the values obtained using ultrasonic sensors. The results obtained using the DIC system, MTS, and strain gauges demonstrate the computational efficiency, experimental reliability, and numerical accuracy of the proposed DIC algorithm.

\section{Acknowledgments}

This research was funded by the Ministry of Science and Technology (MOST) of the Republic of China, Taiwan, under Contract Nos. 108-2622-E-027-021-CC3, 107-2218-E-027018, and 108-2218-E-027-017.

\section{References}

1 K. Zhou and Z. Wu: Eng. Struct. 141 (2017) 184.

2 M. Malesa, K. Malowany, J. Pawlicki, M. Kujawiska, P. Skrzypczak, A. Piekarczuk, T. Lusa, and A. Zagorski: Eng. Fail. Anal. 69 (2016) 122.

3 J. Zhao: Opt. Eng. 55 (2016) 124104.

4 M. A. Caminero, M. Lopez-Pedrosa, C. Pinna, and C. Soutis: Appl. Compos. Mater. 21 (2013) 91.

5 G. Ruocci, C. Rospars, G. Moreau, P. Bisch, S. Erlicher, A. Delaplace, and J.-M. Henault: Strain 52 (2016) 503.

6 S. W. Khoo, S. Karuppanan, and C. S. Tan: Metrol. Meas. Syst. 23 (2016) 461.

7 K. Genovese, L. Cortese, M. Rossi, and D. Amodio: Opt. Laser Eng. 82 (2016) 127.

8 J.-C. Passieux, F. Bugarin, C. David, J.-N. Périé, and L. Robert: Exp. Mech. 55 (2014) 121.

9 B. Pan and L. Tian: Opt. Eng. 54 (2015) 034106.

10 X. Xu, Q. Zhang, Y. Su, Y. Cai, W. Xue, Z. Gao, Y. Xue, Z. Lv, and S. Fu: Exp. Mech. 57 (2017) 831.

11 X.-Y. Liu, Q.-C. Tan, X. Lei, G.-D. Liu, J.-Y. Liu, J. Y. Liu, X. Yang, and C.-Y. Wang: Opt. Laser Technol. 44 (2012) 1060.

12 J. P. Lewis: Vision Interface 10 (1995) 120.

13 K. Briechle and U. D. Hanebeck: Opt. Pattern Recognit. 4387 (2001) XII.

14 C. Y. Chang and C. C. Ma: Mech. Syst. Signal. Process. 92 (2017) 293.

15 B. Pan, A. Asundi, H. Xie, and J. Gao: Opt. Laser Eng. 47 (2009) 865. 